\title{
From star-disc encounters to numerical solutions for a subset of the restricted three-body problem (Corrigendum)
}

\author{
Andreas Breslau, Kirsten Vincke, and Susanne Pfalzner \\ Max-Planck-Institut für Radioastronomie, Auf dem Hügel 69, 53121 Bonn, Germany \\ e-mail: abreslau@mpifr.de \\ A\&A 599, A91 (2017), DOI: 10.1051/0004-6361/201526068 \\ Key words. methods: numerical - protoplanetary disks - gravitation - planets and satellites: dynamical evolution and stability - \\ scattering - errata, addenda
}

After publication of Breslau et al. (2017) we noticed a mistake in the plot script used to produce Figs. $2 \mathrm{~d}$, 3d, and $5 \mathrm{~d}$. These images should have shown the final semi-major axes of the bound particles relative to the radii of their initial orbits depending on their virtual pericentre positions (VPPs). Due to an incorrect multiplication with $(1-e)$, the images instead showed the final periapsides of the bound particles relative to their initial orbital radii. In this erratum we present the corrected images (see Fig. 1).

Because the original images were not correct, the text in the paper also described the final periapsides and not the final semimajor axes. The correct descriptions for the final semi-major axes are presented below.

In Fig. 1a the final semi-major axes are shown for a mass ratio of $m_{12}=0.1$. The final semi-major axes have large values $(\gtrsim 2)$ along the regions where the particles become unbound. With increasing distance from these regions, the values of the final semi-major axes decrease. This decrease with distance is steeper in the regions where the particles move ahead of the perturber (e.g. around $(1,-3)$ and $(1,2))$ and is shallower where the particles move behind the perturber (e.g. around $(-1,4)$ and $(3,1))$. Along the negative $\mathrm{x}$-axis the values of the final semimajor axes are close to the radii of the initial orbits (green region). Above the negative $x$-axis there is a larger, elliptical region where the final semi-major axes are also larger even though the region is far away from the region where the particles become unbound. Ahead of the departing branch of the perturber's interaction orbit there is a wider band where the final semi-major axes are much smaller than the radii of the particle's initial orbits.
For consistency with the paper, we describe next the corrected image for a mass ratio of $m_{12}=20.0$ (see Fig. 1b). For the particles which remain bound to the host, the difference is not large. The particles that are captured by the perturber have, in general, final semi-major axes which are much larger (factor z) than the radii of their initial orbits around the host.

For the mass ratio of $m_{12}=1.0$ the difference in the images is larger. Most particles that remain bound to the host or are captured by the perturber, have final semi-major axes which are smaller than the radii of their initial orbits. In contrast, almost all particles with VPPs in the second quadrant (see Fig. 1c) have finally semi-major axes that are more than twice as large as the radii of their initial orbits. This region is a continuation of the region where the particles are centrifugally removed. Here, the energy gain is just not enough to become unbound.

Due to the changed figures, one sentence in the Summary also has to be adjusted. For the low-mass case $\left(m_{12}=0.1\right)$ the summary of the effect on the particles that remain bound reads now: The closer to the perturber orbit, the higher the final eccentricities and the larger the semi-major axes of the particles that remain bound to the host.

All other results and conclusions in the paper remain unaffected.

\author{
References \\ Breslau, A., Vincke, K., \& Pfalzner, S. 2017, A\&A, 599, A91
}


(a)

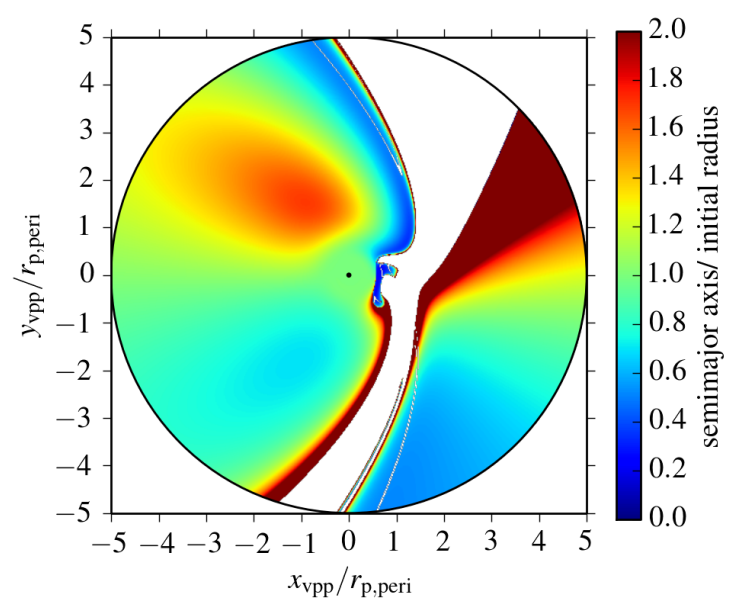

(b)

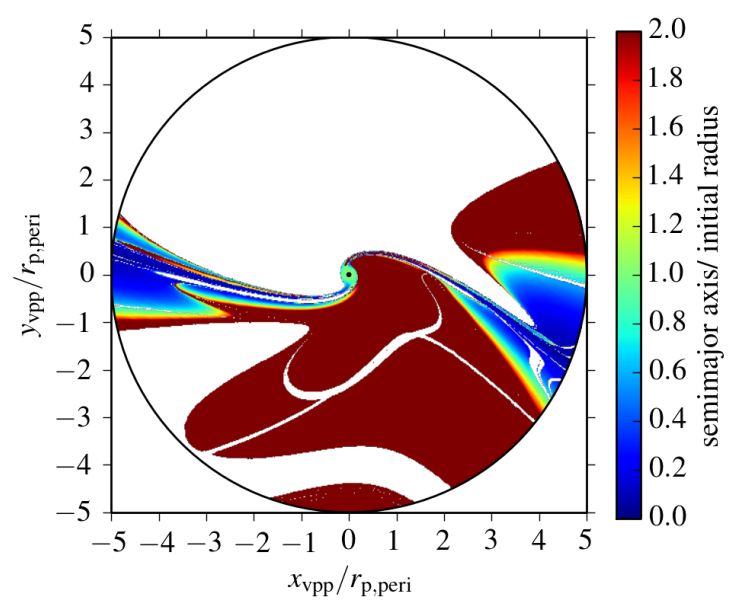

(c)

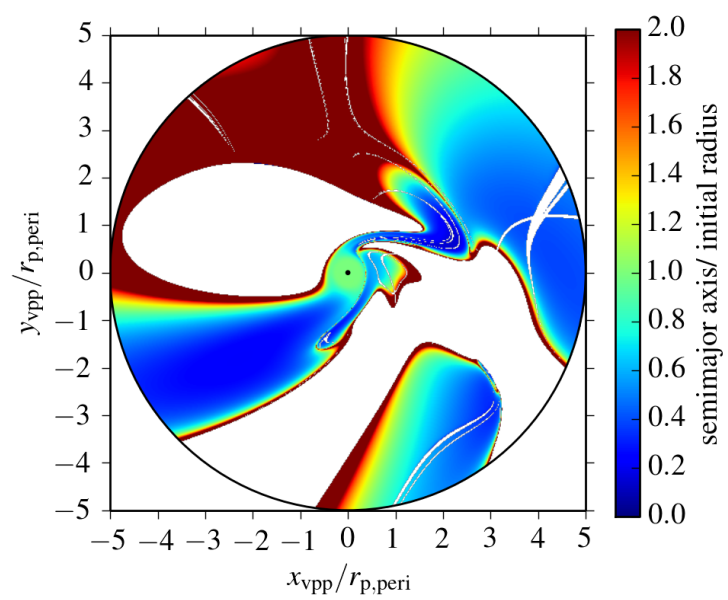

Fig. 1. Maps of the final semi-major axes of the bound particles relative to the radii of their initial orbits depending on their VPPs for the region $r_{\mathrm{vpp}} / r_{\text {p.peri }}<5$ for the mass ratios $m_{12}=0.1(a), m_{12}=20.0(b)$, and $m_{12}=1.0(c)$. 